\title{
A Hybrid PSO-SVM Based Method for Degradation Process Prediction of Reciprocating Seal
}

\author{
Madhumitha Ramachandran ${ }^{1}$, Jon Keegan ${ }^{2}$, and Zahed Siddique ${ }^{3}$ \\ ${ }^{1}$ School of Industrial and Systems Engineering, University of Oklahoma, Norman, OK, 73019, USA \\ madhumitha23@ou.edu \\ ${ }^{2,3}$ School of Aerospace and Mechanical Engineering, University of Oklahoma, Norman, OK, 73019, USA \\ jon.r.keegan-1@ou.edu \\ zsiddique@ou.edu
}

\begin{abstract}
Reciprocating machines such as piston pumps, compressors and internal combustion engines are widely used in a wide range of manufacturing industries including automotive, aerospace, paper, oil and gas, etc. Reciprocating seal located directly on the rod/piston of a reciprocating equipment is used for preventing leakage and reducing wear between two parts that are in relative motion. Seal failure is one of the foremost causes of breakdown of reciprocating machinery and such a failure can be catastrophic, resulting in costly downtime and large expenses. Assessment of reciprocating seal is extremely important in the manufacturing industry to avoid fatal breakdown of reciprocating equipment and machines. Prediction of time series using predictive maintenance practices and tools to estimate the evolution of the future condition of the system is of great interest to the operators for taking timely and appropriate maintenance decisions. In this paper, we have built and trained a hybrid PSO-SVM model to predict the degradation process of reciprocating seals. Particle swarm optimization is used to optimize the penalty factor and kernel parameter of SVM model. Controlled experiments are designed and performed, and data collected from a dedicated experimental set-up is used to validate the proposed approach.
\end{abstract}

\section{INTRODUCTION}

Dynamic seals such as reciprocating seals are used to separate or retain fluids, pressure and remove contaminants. Failure of reciprocating seal is one of the foremost causes of breakdown of reciprocating machinery and can lead to catastrophic consequences, resulting in costly downtime and large expenses. Replacing a seal after its failure can be extremely expensive, while replacing the seal much earlier before its failure may lead to lower life utilization.
Figure 1 shows the cross-section of reciprocating seal on a rod within reciprocating machines. Material characteristics, amount of seal compression, surface irregularities, inadequate lubrication, fluid contamination, seal size are some of the factors that influence a dynamic seal failure (Center, 1992). The seal wear due to friction between the seal and piston/rod results in increased friction force and excessive leakage.

Predictive model to estimate the evolution of the future condition of seal will result in reduced downtime and maintenance cost, and improved maintenance and logistic planning. In the last decade, numerous physics-based prediction methods, which demands direct estimation of physical parameters such as material, seal and mechanical properties have been proposed by researchers (Salant, Maser, \& Yang, 2007; Yang \& Salant, 2008) has been developed to assess the degradation of polymeric seals. Also, physicsbased approaches are very specific to the material and geometry of the system and requires a new model with any change in the system. Whereas, data-driven based predictive method utilizes indirect measurements using sensor technology and computational capabilities to monitor the current running condition of seals. This technique is primarily applied to metallic parts such as bearings, gear box, etc. and there is a need for research on data-driven based predictive method that can be applied to engineering systems such as polymeric elastomer seals.

In our previous studies, we have developed a data-driven framework utilizing friction torque of rotary seals to classify the running condition of rotary seals (Ramachandran \& Siddique, 2018a, 2018b, 2019b). In this study, we aim to predict the degradation of reciprocating seals based on friction force signal using Support Vector Machines (SVM). To validate our approach, run-to-failure test was conducted

Madhumitha Ramachandran et al. This is an open-access article distributed under the terms of the Creative Commons Attribution 3.0 United States License, which permits unrestricted use, distribution, and reproduction in any medium, provided the original author and source are credited. 
to capture the performance degradation of reciprocating seal using a dedicated reciprocating test set-up.

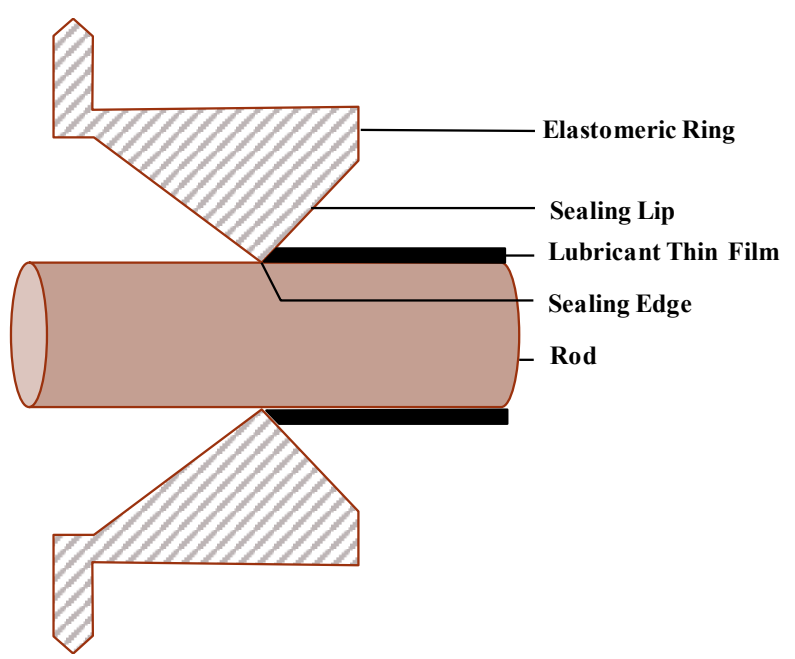

Figure 1. Cross-section of a reciprocating seal on a reciprocating rod

Support Vector Machines has shown to be successful in studying degradation of components because of its performance modeling and generalization attributes, when compared to widely used methods including neural network (Caesarendra, Widodo, \& Yang, 2011; Sun, Zhang, \& He, 2011). SVM was found to be successful in predicting the degradation of bearing, batteries and other electronic and mechanical components. Dong and Luo (2013) developed a PCA based optimized LS-SVM model to predict the degradation process of bearings. Sun et al. (2011) used the SVM-based model to predict bearing degradation by using few failed bearing data. They allocated different weights to different bearings and the information is fused to predict the degaradtion of test bearing. Park and Jeong (2013) used recurive SVM to predict the degradation of secondary rechargebale battery. Guo, Ma, Xiao, and Tian (2012) developed a PSO-SVM model to diagnose faults in electronic system. $\mathrm{Xu}, \mathrm{Wu}, \mathrm{Guo}$, and $\mathrm{Hu}$ (2010) used LS-SVM to predict the life of barrels of tank guns. According to Huang, Wang, Li, Zhang, and Liu (2015) applications of SVM in building degradation modes are limited to componenets such as bearings and batteries; and they stress the need for research on applications of SVM in predicting the degradation of other key componenets.

The most challenging aspect of SVM is tuning the hyperparameters of SVM during the training phase, which requires solving an optimization problem. In our previous paper we implemented grid search method to optimize the SVM parameters (Ramachandran \& Siddique, 2019a) and in this paper, we have implemented phase space reconstruction to determine the input and output vectors of the prediction model and particle swarm optimization, a meta-heuristic approach, to optimize the SVM parameters to improve the prediction accuracy.
The remainder of this paper is structured as follows: description of experimental test set-up, data collection, phase space reconstruction, particle swarm optimization and support vector machines are explained in detail in experimental methods section; results of our approach are discussed in results and discussion section; study conclusions are summarized in conclusion section.

\section{EXPERIMENTAL METHODS}

\subsection{Test Set-up}

Oscillating seal test fixture mimics the mechanical dynamics of a conventional positive displacement piston pump. Mechanics of interest are isolated to the reciprocating/shuffling motion of a piston or rod within a stationary bore. There exist two common methods of sealing fluids within dynamic reciprocating applications: piston seals and rod seals. Interest here will be to characterize the performance of rod seals. A main pressure chamber encapsulates an interchangeable core. This modular core can be made with many distinct seal geometry gland dimensions for the purpose of testing a variety of polymer seals with differing cross sections. Experimental runs depicted here concern standard AS568, size 214 circular cross section Orings. Reciprocating through this modular core lies a precision ground rod of desired surface roughness 16RMS. Reciprocating rod is driven by a Baldor 3HP M3611T-9 alternating current three-phase induction motor affixed to a Baldor 10:1 gear reduction transmission whose output shaft turns a cam-arm (as shown in Figure 2). This cam-arm pushes and pulls the rod through one reciprocation for every revolution of gearbox output shaft. Stroke length of reciprocation is fixed at 10.5 inches and is determined by length of cam-arm.

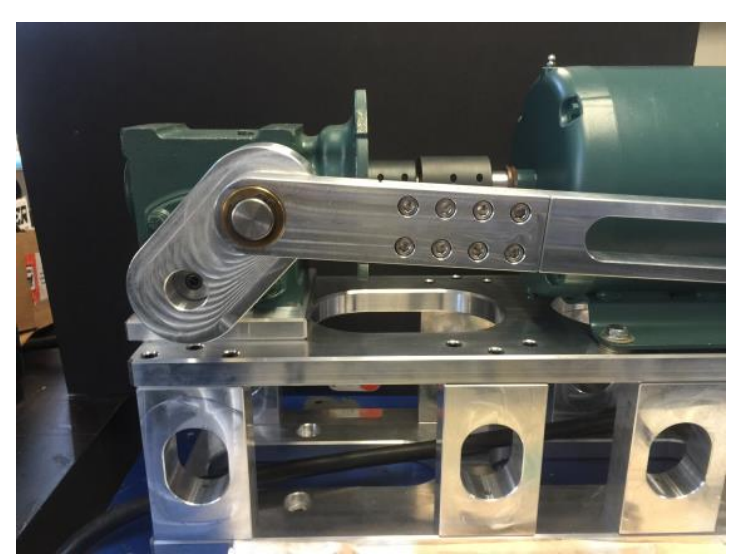

Figure 2. Test set-up showing induction motor, gearbox cam-arm diving reciprocating rod motion

Baldor 3HP induction motor is driven by an ABB ACS15003U-09A8-2 3HP DRIVE. This variable frequency drive gives motor speed and torque control by varying the frequency and voltage supplied to the motor by main power 
lines. Frequency ranges from $0-60 \mathrm{~Hz}$, for out motor $0 \mathrm{~Hz}$ equates to ORPM and $60 \mathrm{~Hz}$ equates to $1750 \mathrm{RPM}$.

A fixed motor speed and a fixed cam-arm length will parameterize experimental runs expressed in this paper. Motor speed at gearbox output shaft has been fixed at 30 RPM and cam-arm length set to 5.5 inches. Recall that for every separate revolution of the motor gearbox output shaft, the cam-arm moves through one revolution, which translates circular motion into linear motion, hence, the title cam-arm. This means that for every separate revolution, the cam-arm moves a total of 11 inches, 5.5 inches of stroke in compression and 5.5 inches of stroke in tension. For our purposes, with the fixed parameters specified above, one reciprocation equates to 330 inches of rod travel per minute.

To keep linear motion consistent and without binding, Thompson linear guide and ball bearing carriage are mated to reciprocating rod. As power is transmitted from motor and cam-arm, opposing end of cam-arm pivots on a shuttle specifically made to accept power transmission. This shuttle has been designed to transmit cam-arm movement into an Omega Engineering LC203-1K bi-directional load cell. As shuttle pushes reciprocating rod through 'compression cycle' and pulls reciprocating rod through 'tension cycle' real time force data is acquired. Overview of reciprocating rod, load cell and shuttle can be seen in Figure 3.

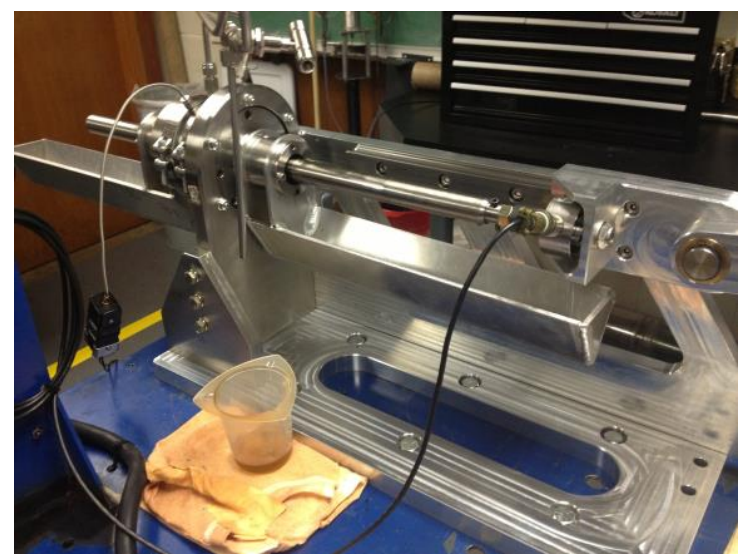

Figure 3. Test set-up showing testing chamber, reciprocating rod, load cell and shuttle

Main pressure chamber and seals are energized through the hydraulic pressurization of Aero Shell 560 Turbine oil. Considering the 'leak rate' of seals to be of interest it is required to keep system at a constant pressure elimination one variable in the experimental run. To counteract the transient effects of mechanical cycling of a hydraulic pump, a specialty hydraulic piston accumulator with active piston position feedback is used to transmit high-pressure nitrogen to highpressure hydraulic oil.

Hydraulic piston accumulator with live piston positioning allows for active tracking of displaced fluid through system, or 'leaked fluid while system is kept at constant pressure. The hydraulic piston accumulator accomplishes this by implementing an MTS GHT0060UFD21V0 liner variable differential transducer to actively track position of piston within bore of accumulator.

Special design of experiment conditions requires simulating real operating conditions of seals within componentry exposed to harsh temperature conditions. Test chamber assembly has been outfitted with large diameter 240volt mica heating bands. Heating bands are controlled with Omega Engineering iSeries programmed proportional integral differential (P.I.D.) temperature controller. This controller allows precise temperature control $+/-1^{\circ} \mathrm{C}$ for all experimental runs conducted thus far up to $200^{\circ} \mathrm{C}$.

\subsection{Data Collection}

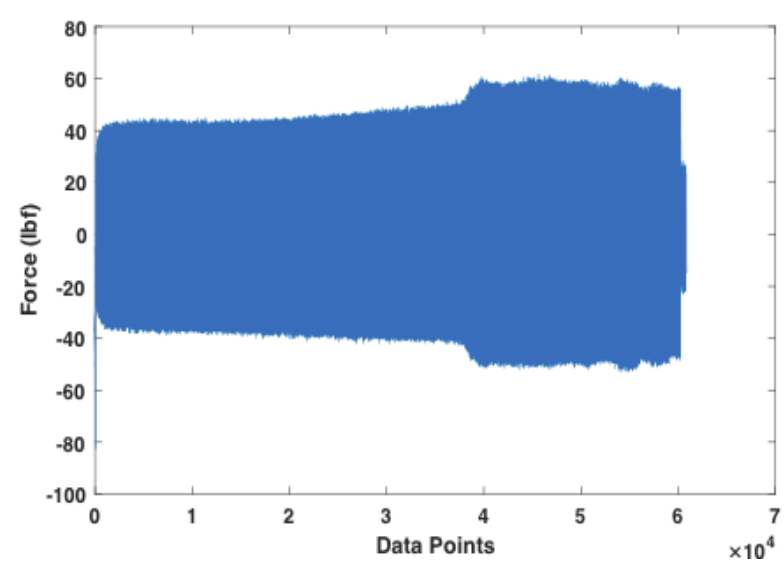

Figure 4. Run-to-failure test of seal-1

Real time data from all transducers is acquired through the utilization and combination of National Instruments LabVIEW in conjunction with National Instruments USB XSeries 6341 Multifunction I/O device. Device acquires data through high speed TDMS streaming and functions as a controller for all peripheral componentry. Eight run-tofailure tests at $150^{\circ} \mathrm{C}, 1500$ PSI are performed to collect information on the force required to move reciprocating rod some unit length per unit time. 20 data points are sampled every second. Figure 4 shows the run-to-failure test of seal-1 of data length of 60,370. For a better visualization, a segment of run-to-failure force signal is shown in Figure 5. Figure 5 shows two regions of interest: Region 1 will overview force vs. time while reciprocating rod is in tension and Region 2 will overview Force vs. Time while rod is in compression. Considering these two regions to be mirrors of each other, we will simply limit our discussion to Region 1 knowing that Region 2 is the inverse. Within Region 1 we see a positively oriented parabolic trend from point A-B. If we consider this region to be in simple tension, then we would expect the force graph to be almost perfectly flat therefore why is it not? Experiments have led us to believe the force graph is affected by two things: first is rooted in the mechanics of this system. 
There exists some small acceleration and deceleration at the leftmost and rightmost limit of the reciprocating stroke. This causes upon switching from tension to compression $\mathrm{B}-\mathrm{B}^{*}$ or compression to tension $\mathrm{A}^{*}$-A some momentary 'jerk' resulting in a transient spike in force graph. Second comes from the idea of seal wiping. This phenomenon exists when the seal physically wipes excess lubricating film from the reciprocating rod. At leftmost and rightmost stroke limits the seal has less fluid to wipe away resulting in a not fully lubricated seal which in turn increases force seen at stroke limits. It takes around 3.90 seconds to complete a tension and compression cycle.

\subsection{Phase Space Reconstruction}

Following feature extraction, phase space reconstruction based on Takens embedding theorem is used to construct a set of vectors whose components are lagged version of time series (Takens, 1981). Let $X_{t}=\left\{x_{1}, x_{2}, \ldots, x_{N}\right\}$ be a univariate time series of length $N$ and $y_{i m}=\left\{x_{i}, x_{i+\tau}, \ldots, x_{i+(m-1) \tau}\right.$, where $i=1,2, \ldots, N_{m}, N_{m}=N-(m-1) \tau$ is the length of reconstructed vector, $\tau$ is the time delay, $m$ is the embedding dimension. In 1986, Fraser and Swinney found

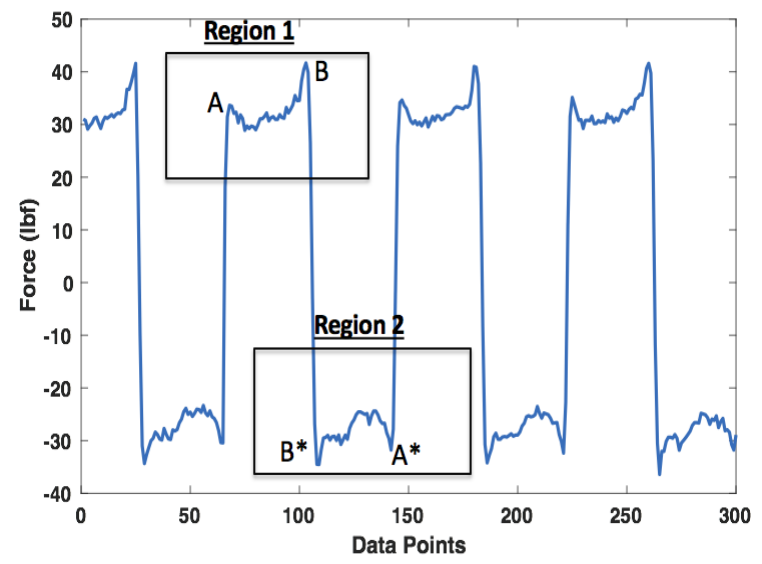

Figure 5. A segment of force signal showing tension and compression cycle

the concept of time delay for embedding a univariate time series (Fraser \& Swinney, 1986). The time delay $\tau$ is estimated using average mutual information which is a nonlinear generalization of the autocorrelation function. The average mutual information estimates how much a time series $x(t)$ can provide information on another time series $x(t+\tau)$ and is given by:

$$
I(x(t), x(t+\tau))=\sum_{i j} p_{i j}(\tau) \log \left(\frac{p_{i j}(\tau)}{p_{i} p_{j}}\right)
$$

Where, $p_{i}$ is the probability that the time series $x(t)$ is in the bin $i$ of the histogram constructed from data points in series $x(t), p_{j}$ is the probability that the time series $x(t+\tau)$ is in the bin $j$ of the histogram constructed from data points in series $x(t+\tau)$ and $p_{i j}$ is the probability that the time series $x(t)$ is in the bin $i$ and $x(t+\tau)$ is in the bin $j$. The optimal time delay is estimated using the position of the first minimum of average mutual information function.

The embedding dimension $m$ is determined using CAO method (Cao, 1997) and is defined as:

$$
a(i, m)=\frac{\left\|y_{i}(m+1)-y_{i}^{n n}(m+1)\right\|}{\left\|y_{i}(m)-y_{i}^{n n}(m)\right\|}
$$

Where, $\|\cdot\|$ is the Euclidean distance given by the maximum norm. $y_{i}(m)$ is the $i^{\text {th }}$ reconstructed vector and $y_{i}^{n n}(m+1)$ is the nearest neighbor of $y_{i}(m)$ in embedding dimension $m$. The mean value of all $a(i, m)$ is defined as:

$$
E(m)=\frac{1}{N-m \tau} \sum_{i=1}^{N-m \tau} a(i, m)
$$

$E(m)$ depends on dimension $m$ and lag time $\tau$ and its variation from $m$ to $m+1$ is given by a parameter $E_{1}$ :

$$
E_{1}(m)=\frac{E(m+1)}{E(m)}
$$

$E_{l}(m)$ stops increasing when $m$ is greater than some value $m_{o}$. $E_{l}(m)$ increases slowly or stops changing if $m$ is significantly large and to overcome this CAO introduced another quantity $E_{2}(m)$ which is given by:

$$
E_{2}(m)=\frac{E^{*}(m+1)}{E^{*}(m)}
$$

Where

$$
E^{*}(m)=\frac{1}{N-m \tau} \sum_{i=1}^{N-m \tau}\left|x_{i+m \tau}-x_{i+m \tau}^{n n}\right|
$$

Therefore, $E_{l}(m)$ is estimated to determine the minimum embedding dimension of time series and $E_{2}(m)$ is used to distinguish the deterministic signals from stochastic signals

\subsection{Particle Swarm Optimization}

Particle swarm optimization, a population based intelligent algorithm proposed by Kennedy and Eberhart in 1995 is used to optimize SVM regression parameters (Eberhart \& Kennedy, 1995). PSO simulates the behavior of swarms in solving optimization problems iteratively because of its simplicity, fast convergence and high performance. PSO is based on a set of particles and their coordinates represent a potential solution of an optimization problem. The position and velocity of $i^{t h}$ particle in a population with $N$ particles in a $d$ - dimensional space is given by $X_{i}(t)=\left(x_{i 1}(t)\right.$, $\left.x_{i 2}(t), \ldots, x_{i D}(t)\right)$ and $V_{i}(t)=\left(v_{i 1}(t), v_{i 2}(t), \ldots, v_{i D}(t)\right)$. PSO algorithm updates the velocity and position of the particles at iteration $t+1$ using the following equations.

$$
\begin{aligned}
v_{i d}(t+1)= & \omega v_{i d}(t)+c_{1} r_{1}\left(p_{i d}(t)-x_{i d}(t)\right)+ \\
& c_{2} r_{2}\left(p_{g d}(t)-x_{i d}(t)\right)
\end{aligned}
$$




$$
x_{i d}(t+1)=x_{i d}(t)+v_{i d}(t+1)
$$

Where $i=1,2, \ldots, N$ denotes particles of population size $N$ $d=1,2, \ldots, D$ denotes dimension of search space with $\mathrm{D}$ dimensions

$\mathrm{t}=$ current iteration with the number of iterations $\mathrm{T}$

$\omega=$ inertia weight to balance the local and global search abilities of particles

$c_{1}, c_{2}=$ learning factors

$r_{1}, r_{2}=$ random functions with uniform distribution of $(0,1)$

$v_{i d}(t)=$ velocity of the $i^{t h}$ particle in $t^{t h}$ iteration

$x_{i d}(t)=$ position of the $i^{t h}$ particle in $t^{t h}$ iteration

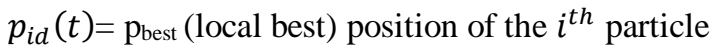

$p_{g d}(t)=g_{\text {best }}($ global best $)$ position of the particle

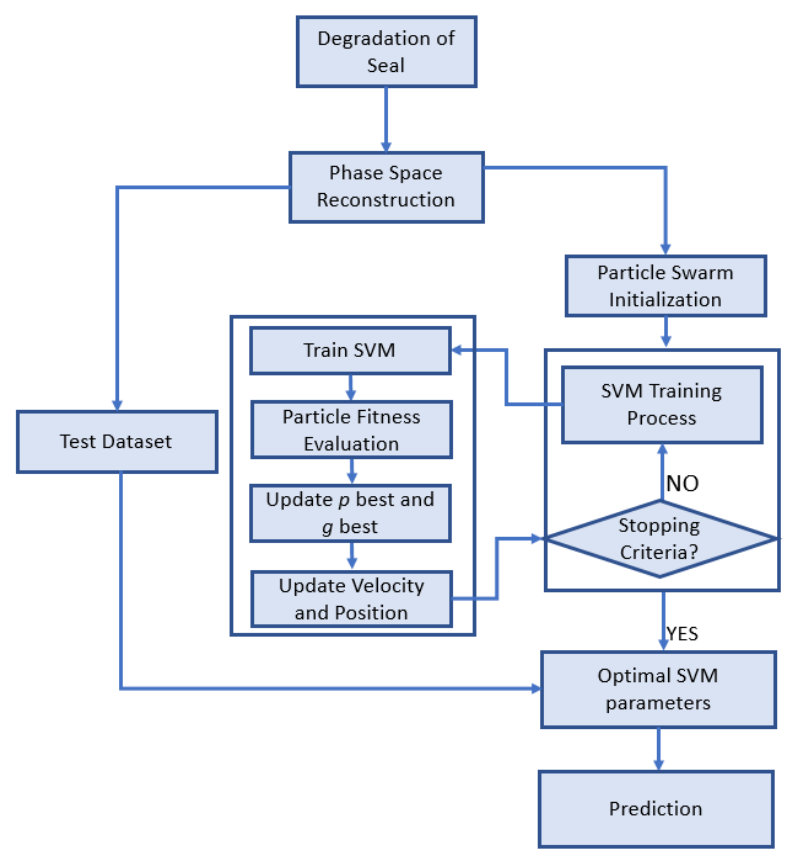

Figure 6. Flowchart showing the PSO-SVM Procedure

The flowchart of optimizing SVM regression parameters: penalty factor $C$ and kernel function parameter $g$ based on PSO is shown in Figure 6 and the procedure is given below:

1. Randomly initialize population size, learning factors, inertia weight, random functions, position and velocity of each particle. Initialize the SVM regression parameters and set the termination condition.
2. SVM model is trained with the initialized parameters and the fitness value of each particle is calculated using the PSO fitness function given in Eq. (17)

3. The $p_{\text {best }}$ and $g_{\text {best }}$ values are adjusted based on the particle fitness value and the velocity and position of the particles are updated using the equation given in Eq. (7) and (8).

4. The SVM parameters are updated and the model is retrained is retrained using the PSO fitness function. Step 3 and step 4 is continued until the termination condition is satisfied.

5. When the termination condition is met, optimal parameters $C$ and $\gamma$ of SVM regression is found.

6. Retrain SVM regression with the optimal values and tested on the SVM regression.

\subsection{Support Vector Regression}

Support Vector Machine based on statistical learning theory is put forward by Vapnik in 1995. Given a set of training data $\left\{\left(X_{1}, y_{l}\right),\left(X_{2}, y_{2}\right), \ldots,\left(X_{l}, y_{l}\right)\right\}, X_{i} \in R^{m}, y_{i} \in R$, where $y_{i}$ is the dependent variable for a given set of independent variable $X_{i}$, SVM generalization to Support Vector Regression (SVR) is performed by defining an $\varepsilon$-insensitive region, inside which errors are ignored. SVR formulates it as an optimization problem, where the objective is to find the flattest region (means to have a small $\omega$ ) that contains most of the training instances while minimizing the prediction error (distance between the predicted and actual values). The regression in terms of constrained optimization problem can be written as:

$$
\begin{aligned}
& \text { minimize } \frac{1}{2}\|\mathcal{W}\|^{2}+C \sum_{i=1}^{l}\left(\xi_{i}+\xi_{i}^{*}\right) \\
& \text { subject to } y_{i}-\mathcal{W} x_{i}-b \leq \varepsilon+\xi_{i} \\
& \mathcal{W} x_{i}+b-y_{i} \leq \varepsilon+\xi_{i}^{*} \\
& \xi_{i}, \xi_{i}^{*} \geq 0
\end{aligned}
$$

where $\frac{1}{2}\|\mathcal{W}\|^{2}$ is the weight vector, $y_{i}$ is the desired value and the tuneable parameter $C$ is the penalty factor that determines the trade-off between the flatness and the extent to which deviations larger than $\varepsilon$ are tolerated. The loss function defined is non-differentiable due to the absolute value in function, so the positive slack variables $\xi_{i}, \xi_{i}^{*}$ are introduced to account for the errors in points that lie outside the $\varepsilon$-insensitive region. Applying Lagrangian multiplier, this problem is converted into dual optimization problem as follows:

$$
\begin{aligned}
& \max \left[-\frac{1}{2} \sum_{i, j=1}^{n}\left(\alpha_{i}-\alpha_{i}^{*}\right)\left(\alpha_{j}-\alpha_{j}^{*}\right)\left(x_{i}, x_{j}\right)-\right. \\
& \left.\varepsilon \sum_{i=1}^{n}\left(\alpha_{i}-\alpha_{i}^{*}\right)+\sum_{i=1}^{n}\left(\alpha_{i}-\alpha_{i}^{*}\right)\right] \\
& \text { Subject to } \sum_{i=1}^{n}\left(\alpha_{i}-\alpha_{i}^{*}\right)=0 \text { and } \alpha_{i}, \alpha_{i}^{*} \in[0, c]
\end{aligned}
$$


Where $\alpha_{i}, \alpha_{i}^{*}$ are lagrangian multipliers. For nonlinear regression, SVM uses a kernel function. It performs by mapping the data $\mathrm{X}$ into a high dimensional feature space by non-linear mapping and then performing regression in the feature space. Using the kernel function $K\left(x_{i}, x_{j}\right)$, the regression function is as follows:

$$
f(x)=\sum_{i, j=1}^{n}\left(\alpha_{i}-\alpha_{i}^{*}\right)\left(\alpha_{j}-\alpha_{j}^{*}\right) K\left(x_{i}, x_{j}\right)+b
$$

Where $K\left(x_{i}, x_{j}\right)$ is a kernel function. There are different kernel functions such as Linear, Polynomial, Radial Basis Function and Sigmoid. In this paper, RBF function is utilized, and it is given by:

$$
K\left(x_{i}, x_{j}\right)=\exp \left(-\gamma\left\|x_{i}-x_{j}\right\|^{2}\right),(\gamma>0)
$$

Where $\gamma$ is the RBF kernel parameter.

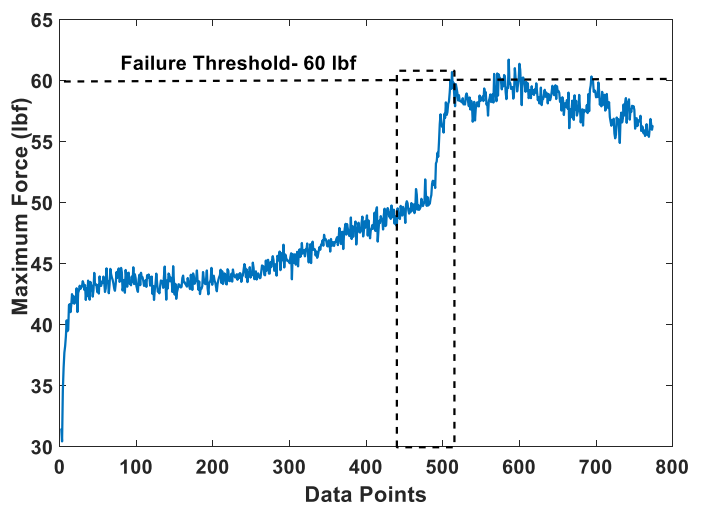

Figure 7 Degradation trend captured by tension peak with the rectangular box showing the degradation region

\subsection{Performance Criteria}

The performance of the model is evaluated using Mean Square Error (MSE). The MSE is defined as:

$$
M S E=\frac{1}{N} \sum_{i=1}^{N}\left(p_{i}-y_{i}\right)^{2}
$$

where $N$ is the total number of data points, $y_{i}$ is the actual value and $p_{i}$ is the predicted value of the model.

\section{RESULTS AND DISCUSSION}

First, the entire time series is segmented into $\mathrm{n}$ segments and features are extracted from each segments of the time series. For example, length of seal-1 run-to-failure test is 60,372 and it is segmented into 774 segments (774 cycles). Main reason for the reciprocating seal to fail is due to rolling, during which the friction increases leading to increased friction force and leakage.

As the seal degrades, compression peak: $A^{*}$ (maximum force during compression cycle) and tension peak: B (maximum force during tension cycle) shown in Figure 5 increases which results in significant leakage. The increase in tension peak can be observed in Figure 7. It was found that as the tension peak begins to increase and approach a force of 60 poundforce, a significant amount of leakage was observed. This can be observed by comparing Figure $8 \mathrm{a}$ and $8 \mathrm{~b}$ which shows the degradation region (data points 430-515) in terms of tension force and leakage. After the failure threshold, seals start leaking excessively (> $1 \mathrm{~mL} /$ cycle) and are labeled as failed. From Figure 5 we can see that the compression and tension regions are mirror image of each other and therefore, we decided to utilize tension peak to predict the degradation process of reciprocating seals. The tension peak value is extracted from every tension cycles of the raw force signal; this way we reduced the complexity of the raw data while also preserving the characteristics of the original data as much as possible.

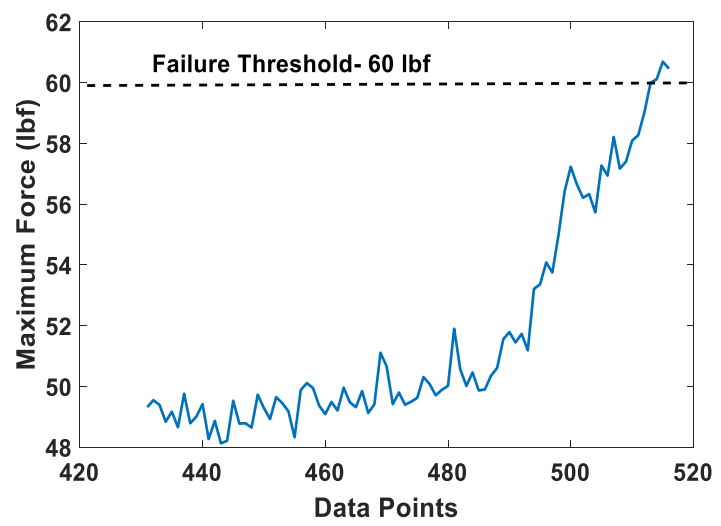

(a)

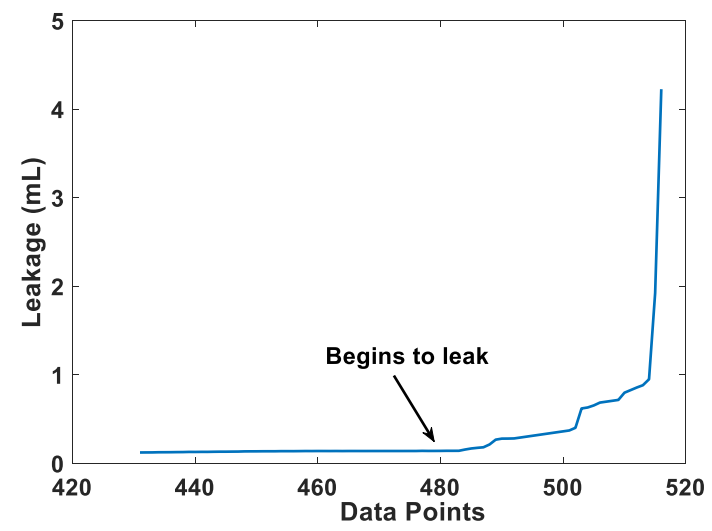

(b)

Figure 8 Degradation region in terms of (a) tension peak and (b) leakage

Out of eight run-to-failure tests, three tests (seal-1, seal-5, seal6) are used to develop the proposed model and the proposed model is tested on the rest. Figure 9 shows the normalized tension peak signal of run-to-failure tests. In order to verify if the tension peak feature would be an ideal indicator of reciprocating seal degradation, Spearman's rank 
correlation coefficient is used to assess the monotonic relationship between the tension peak and run-to-failure time duration of the test. The average correlation coefficient for the training samples is found to be 0.9592 , which indicates tension peak to be a strong indicator of seals degradation process. Average mutual information method is used to estimate the time delay for the phase space reconstruction. It was found that the average mutual information function value reached its first minimum when the time delay was 3 which can be observed in Figure 10.

Then the embedding dimension is selected by the CAO method and Figure 11 shows the selection of the embedding dimension of tension force degradation signal. It can be seen that the optimal embedding dimension for the signal of interest is 7 .

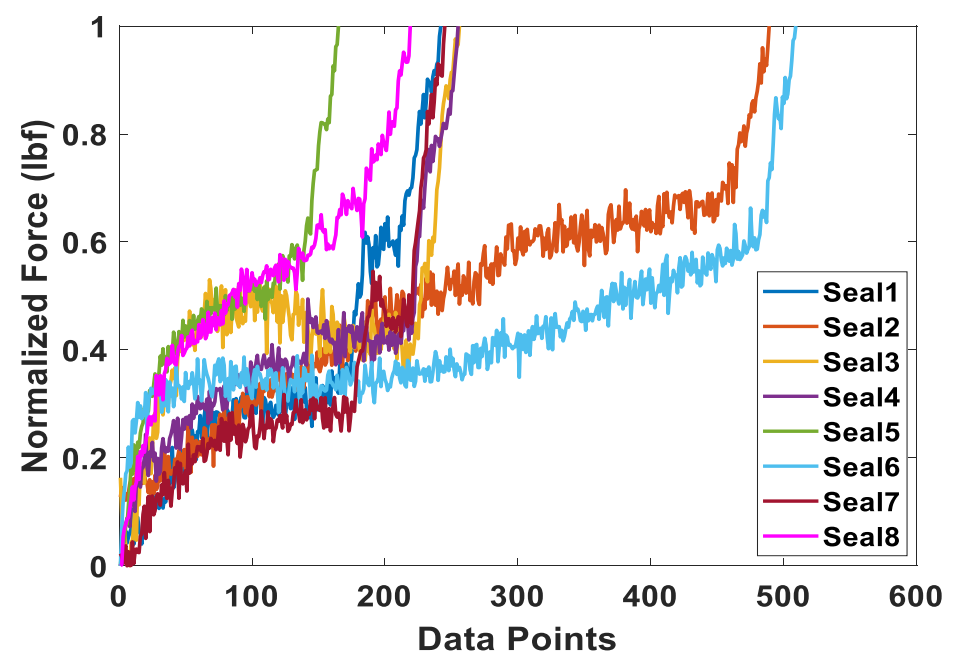

Figure 9 Normalized tension peak signal of run-to-failure test

LIBSVM-3.1-Faruto ultimate 3.1 mode toolbox were used to implement SVM model. The performance of the SVM regression model depends on the penalty factor $C$ and kernel parameter $\gamma$. In this study, an intelligent optimization algorithm: Particle Swarm Optimization was implemented to optimize the SVM parameters. Penalty factor $C$ is an important parameter on which the accuracy of the regression model depends on. When the value of $C$ is too large, the fitting of training samples will be very high, but the generalization ability of the model will be poor whereas when the value of $C$ is too small, optimization process will take very long, and the search will be incomplete leading to a model with poor fitting and low generalization ability. Therefore, the value of $C$ is usually between 0 and 100 to meet the requirements.

\section{Average Mutual Information (AMI)}

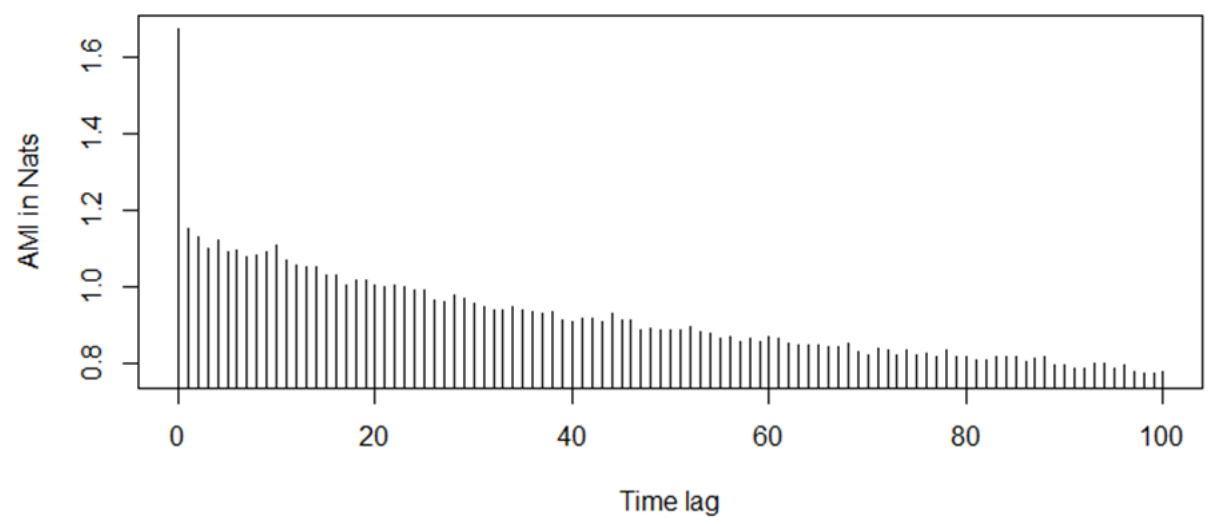

Figure 10 Time delay estimation using Mutual Information Method 


\section{Computing the embedding dimension}

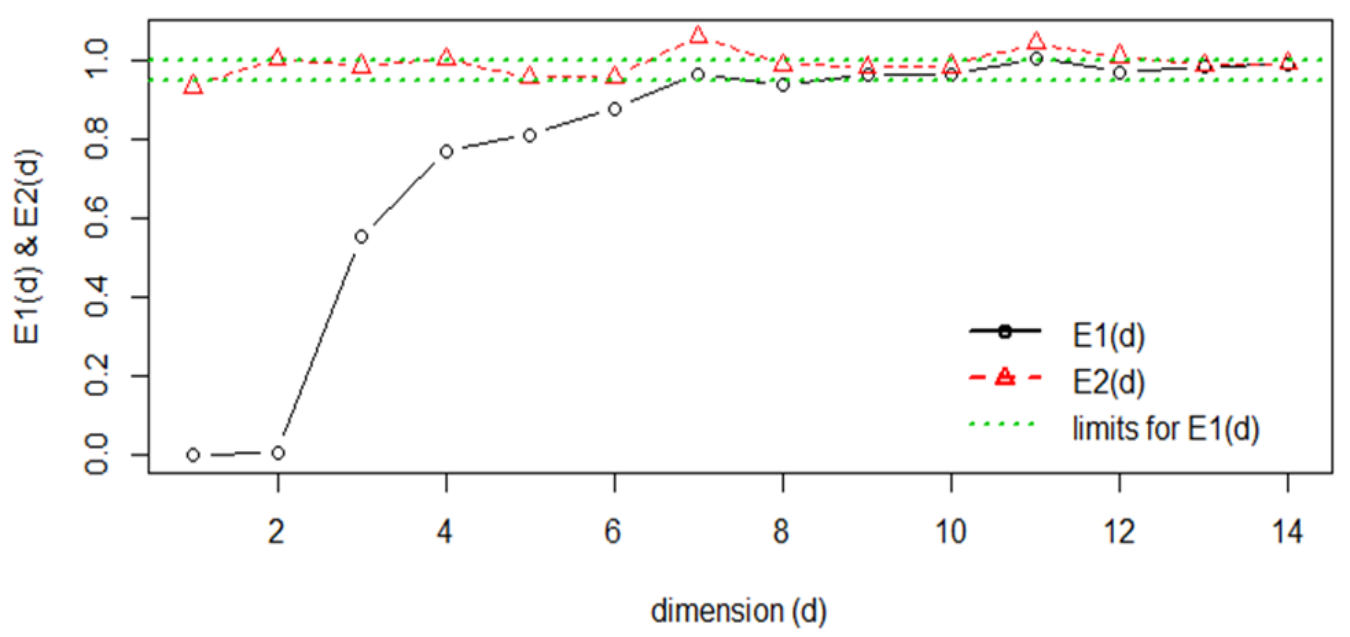

Figure 11 Embedding dimension estimation using $\mathrm{CAO}$ method

The initial parameters of PSO were set to $\omega=0.6, C_{1}=$ $1.5, C_{2}=1.7$, maximum iteration was set to 100 , population size was set to 20 and the penalty factor was in the range of $(0.1,100)$ and kernel parameter was in the range of $(0.01$, 1000). When the termination condition was met the optimal parameters were found to be $\mathrm{C}=15.8136$ and $\gamma=$ 0.01 . The optimal SVM parameters are used to train the SVM model and predict seals degradation. Three run-tofailure tests were used to train the optimized SVM model and the model was tested on the remaining tests. We used the trained model to predict the degradation region (the region before the seal approaches failure threshold) of the remaining tests. The average test MSE of PSO-SVM model on the unseen data was found to be 0.00206 . Figure 12 shows the actual versus the predicted trend of seal-4. It is obvious from Figure 12 that the predicted degradation trend is very close to that of the actual trend.

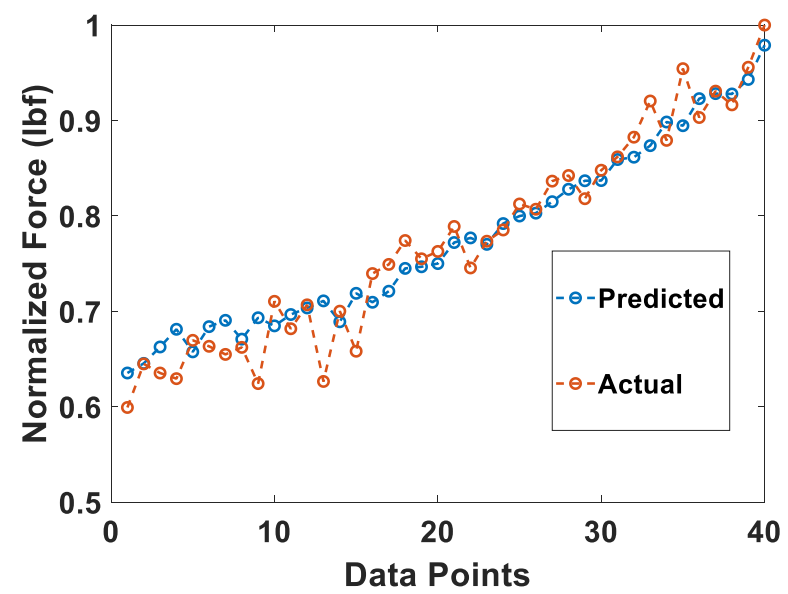

Figure 12 Actual vs predicted for Seal-4 using proposed method
To validate the proposed approach, we used genetic algorithm to optimize SVM parameters and the optimized SVM model was tested on the unseen dataset. Also, we implemented an optimized distributed gradient boosting system: XGBoost to predict the degradation process of seals.

In addition to MSE, we also used Mean Absolute Error to compare the performance of different models. Table 1 shows the performance of the proposed approach compared to other machine learning algorithms ad it is found that the proposed method outperforms other methods used to validate our findings.

Table 1 Performance comparison of different models.

\begin{tabular}{|c|c|c|c|c|c|c|}
\hline \multirow{2}{*}{$\begin{array}{c}\text { Failure } \\
\text { Test }\end{array}$} & \multicolumn{2}{|c|}{ PSO-SVM } & \multicolumn{2}{c|}{ GA-SVM } & \multicolumn{2}{c|}{ XGBoost } \\
\cline { 2 - 7 } & MSE & MAE & MSE & MAE & MSE & MAE \\
\hline Seal-2 & $\mathbf{0 . 0 0 1 0}$ & $\mathbf{0 . 0 2 4 7}$ & 0.0015 & 0.0283 & 0.0048 & 0.0575 \\
\hline Seal-3 & $\mathbf{0 . 0 0 2 9}$ & $\mathbf{0 . 0 4 2 6}$ & 0.0047 & 0.0563 & 0.0032 & 0.0480 \\
\hline Seal-4 & $\mathbf{0 . 0 0 1 7}$ & $\mathbf{0 . 0 3 5 5}$ & 0.0033 & 0.0510 & 0.0078 & 0.0791 \\
\hline Seal-7 & $\mathbf{0 . 0 0 3 1}$ & $\mathbf{0 . 0 4 5 7}$ & 0.0070 & 0.0726 & 0.0038 & 0.0517 \\
\hline Seal-8 & $\mathbf{0 . 0 0 1 5}$ & $\mathbf{0 . 0 3 3 5}$ & 0.0030 & 0.0468 & 0.0022 & 0.0369 \\
\hline Average & $\mathbf{0 . 0 0 2 0}$ & $\mathbf{0 . 0 3 6 4}$ & 0.0039 & 0.0510 & 0.0043 & 0.05464 \\
\hline
\end{tabular}

\section{CONCLSUION}

Data-driven prognostics of dynamic seals such as reciprocating and rotary seals are not very well studied. SVM has found to be effective in predicting the degradation of components such as batteries, bearings and other electronic components. There is little no research on the application of SVM in predicting the future running condition of polymeric components such as seals. In this paper, we have presented a data-driven approach to predict the degradation process of reciprocating seals based on friction force using optimized 
SVM model. The most challenging aspect of tuning hyperparameters in SVM was carried out using particle swarm optimization. The proposed approach was validated using the data collected from an experimental test set-up dedicated to test reciprocating seals. This study shows that SVM model with optimized parameters using PSO is effective in predicting the degradation process of reciprocating seal.

\section{REFERENCES}

Caesarendra, W., Widodo, A., \& Yang, B.-S. (2011). Combination of probability approach and support vector machine towards machine health prognostics. Probabilistic Engineering Mechanics, 26(2), 165173.

Cao, L. (1997). Practical method for determining the minimum embedding dimension of a scalar time series. Physica D: Nonlinear Phenomena, 110(1-2), 43-50.

Center, N. S. W. (1992). Handbook of reliability prediction procedures for mechanical equipment: Carderock Division, Naval Surface Warfare Center.

Dong, S., \& Luo, T. (2013). Bearing degradation process prediction based on the PCA and optimized LSSVM model. Measurement, 46(9), 3143-3152.

Eberhart, R., \& Kennedy, J. (1995). Particle swarm optimization. Paper presented at the Proceedings of the IEEE international conference on neural networks.

Fraser, A. M., \& Swinney, H. L. (1986). Independent coordinates for strange attractors from mutual information. Physical review A, 33(2), 1134.

Guo, Y., Ma, J., Xiao, F., \& Tian, T. (2012). SVM with optimized parameters and its application to electronic system fault diagnosis. Paper presented at the 2012 IEEE Conference on Prognostics and Health Management.

Huang, H.-Z., Wang, H.-K., Li, Y.-F., Zhang, L., \& Liu, Z. (2015). Support vector machine based estimation of remaining useful life: current research status and future trends. Journal of Mechanical Science and Technology, 29(1), 151-163.

Park, J. I., \& Jeong, M. K. (2013). Recursive support vector censored regression for nonparametric lifetime prediction using degradation paths and failure times in accelerated life tests: Citeseer.

Ramachandran, M., \& Siddique, Z. (2018a). A Data-driven, Statistical Feature Based, Neural Network Method for Rotary Seal Prognostics. Journal of Nondestructive Evaluation, Diagnostics and Prognostics of Engineering Systems.

Ramachandran, M., \& Siddique, Z. (2018b, November 9-15, 2018). Statistical Time Domain Feature Based Approach to Assess the Performance Degradation of Rotary Seals. Paper presented at the Proceedings of the ASME 2018 International Mechanical Engineering Conference \& Exposition IMECE 2018, Pittsburgh, Philadelphia, USA.

Ramachandran, M., \& Siddique, Z. (2019a). Degradation Assessment of Reciprocating Seal Using Support Vector Machines. Paper presented at the 14th International Manufacturing Science and Engineering Conference, Erie, Pennsylavania.

Ramachandran, M., \& Siddique, Z. (2019b, June 10-14, 2019). Friction Torque and Leakage Based DataDriven Approach for Rotary Seal Prognostics in Manufacturing Industry. Paper presented at the Proceedings of the 14th International Manufacturing Science \& Engineering Conference MSEC2019, Erie, Pennsylvania, USA.

Salant, R. F., Maser, N., \& Yang, B. (2007). Numerical model of a reciprocating hydraulic rod seal. Journal of tribology, 129(1), 91-97.

Sun, C., Zhang, Z., \& He, Z. (2011). Research on bearing life prediction based on support vector machine and its application. Paper presented at the Journal of Physics: Conference Series.

Takens, F. (1981). Detecting strange attractors in turbulence Dynamical systems and turbulence, Warwick 1980 (pp. 366-381): Springer.

Xu, D., Wu, X.-X., Guo, L., \& Hu, J.-B. (2010). Method of residual life prediction for barrel of tank gun based on LS-SVM. Zhuangjiabing Gongcheng Xueyuan Xuebao(Journal of Academy of Armored Force Engineering), 24(1), 42-44.

Yang, B., \& Salant, R. F. (2008). A numerical model of a reciprocating rod seal with a secondary lip. Tribology Transactions, 51(2), 119-127. 